\title{
Satellite radiometric remote sensing of rainfall fields: multi-sensor retrieval techniques at geostationary scale
}

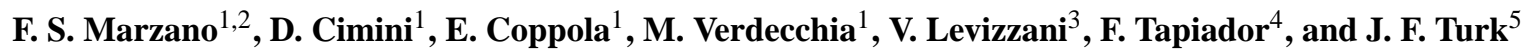 \\ ${ }^{1}$ Centro di Eccellenza CETEMPS, Università dell'Aquila, Via Vetoio - 67010, L'Aquila, Italy \\ ${ }^{2}$ Dipartimento di Ingegneria Elettronica, Università "La Sapienza" di Roma, Rome, Italy \\ ${ }^{3}$ Istituo di Scienze dell'Atmosfera e Clima (ISAC), Consiglio Nazionale delle Ricerche, Bologna, Italy \\ ${ }^{4}$ Instituto de Ciencias Ambientales, Universidad de Castilla-La Mancha (UCLM), Toledo, Spain \\ ${ }^{5}$ Marine Meteorology Division, Naval Research Laboratory (NRL), Monterey, California, USA
}

Received: 11 November 2004 - Revised: 11 July 2005 - Accepted: 13 July 2005 - Published: 22 July 2005

\begin{abstract}
The Microwave Infrared Combined Rainfall Algorithm (MICRA) consists in a statistical integration method using the satellite microwave-based rain-rate estimates, assumed to be accurate enough, to calibrate spaceborne infrared measurements on limited sub-regions and time windows. Rainfall retrieval is pursued at the space-time scale of typical geostationary observations, that is at a spatial resolution of few kilometers and a repetition period of few tens of minutes. The actual implementation is explained, although the basic concepts of MICRA are very general and the method is easy to be extended for considering innovative statistical techniques or measurements from additional space-borne platforms. In order to demonstrate the potentiality of MICRA, case studies over central Italy are also discussed. Finally, preliminary results of MICRA validation by ground based remote and in situ measurements are shown and a comparison with a Neural Network (NN) based technique is briefly illustrated.
\end{abstract}

\section{Introduction}

The problem of using satellite remote sensing data to retrieve rainfall is fairly complicated since presently there is not a single spaceborne platforms which can carry all the suitable instruments to ensure all the properties to the rainfall product (Levizzani et al., 1995; Vicente et al., 1998; Turk et al., 1999). From a meteorological point of view, visible (VIS) and infrared (IR) radiometers can give information on cloud top layers. On the other hand, microwave (MW) radiometers can detect cloud structure and rainrate since MW brightness temperatures (TB's) are fairly sensitive to liquid and ice hydrometeors (Ferraro, 1997). Regarding platforms, Geosynchronous Earth Orbit (GEO) satellites can ensure a cover-

Correspondence to: F. S. Marzano

(marzano@die.uniroma1.it) age with a high temporal sampling, while Low Earth Orbit (LEO) satellites have the advantage to enable the use of microwave sensors, but with a major drawback of low temporal sampling. Therefore, LEO-MW and GEO-IR radiometry are clearly complementary for monitoring the Earth's atmosphere and a highly variable phenomenon such as precipitation.

Statistical integration of satellite infrared and microwave data can be accomplished in several ways (Kummerow and Giglio, 1995; Bellerby et al., 2000; Miller et al., 2001; Marzano et al., 2001). On one hand, there is a choice of what variables (i.e., predictors) to match in order to provide the final product. A possibility is represented by the direct combination of MW TB's and thermal IR (TIR) radiances, having the advantage to exploit the observable information without any post-processing and the disadvantage to request IR and MW measurements matched in space and time. The latter condition is only satisfied a limited number of times in a given area if few LEO platforms are considered thus flaring the statistical significance of any empirically-trained retrieval algorithm. The feasible approach would be that based on physically-based retrieval algorithms which, on the other hand, would need a climatological and microphysical tuning.

In order to avoid these difficulties, one can resort to approaches whose aim is to combine IR measurements and MW-based estimates on a cumulative statistical distribution basis. Indeed, artificial neural network $(\mathrm{NN})$ can be conveniently applied to the same problem dealing with empirically-trained algorithms showing comparable performances (Hsu et al., 1997; Grimes et al., 2003; Tapiador et al., 2004a, 2004b).

In this work, the Microwave Infrared Combined Rainfall Algorithm (MICRA) is presented, based on the statistical integration of collocated GEO-IR and LEO-MW data accomplished on a local scale (Marzano et al., 2003, 2004) and it is compared with a NN-based algorithm: NEREMIS (NEural Rainfall Estimation algorithm using Microwave and Infrared 


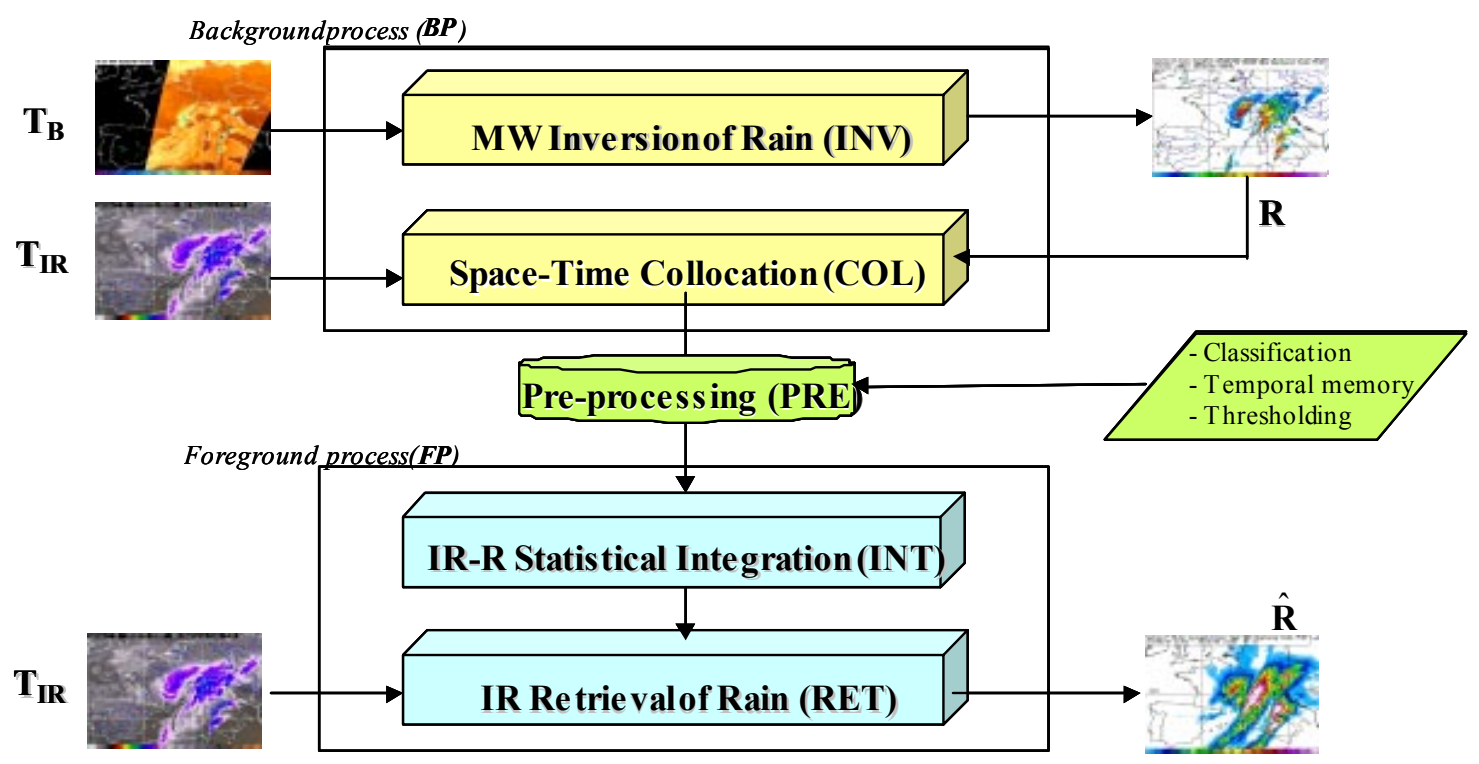

Fig. 1. Schematic sketch of the MICRA block diagram and flow chart.

Sensors). As an application, some case studies on the area of central Italy are discussed, comparing satellite based estimates with ground-based remote (radar) and in situ (rain gauges) observations, in order to demonstrate the potentiality of monitoring precipitation using the proposed techniques.

\section{Microwave infrared combined rainfall algorithm}

The general idea behind the considered integration techniques is to combine the appealing spatial and temporal sampling of IR sensors, mounted on geo-stationary platforms (GEO-IR), with the higher accuracy of passive Low-EarthOrbit Microwave (LEO-MW) methods for rain-rate retrieval. The statistical integration techniques are applied within a procedure which is supposed to run continuously.

This procedure is based on a background process and a foreground process. A schematic sketch of the block diagram and the temporal flow chart of MICRA is shown in Fig. 1, while detailed equations are described in (Marzano et al., 2003; Marzano et al., 2004).

i) The first step of the background process consists in estimating the surface rain-rate from available LEO-MW measurements by means of either empirical retrieval algorithms or inversion schemes based on parametric cloud radiative models (inversion step). This enables the inversion of a set of brightness temperatures (TB) at different frequency and polarization to provide a rainrate product spatially integrated within the nominal area A.

ii) The second step of the background process pursues the combination of LEO-MW sensor data with data coming from GEO-IR sensor in space and time on a global scale (collocation step). This step consists in temporally locating the GEO-IR data within the past few tens of minutes of the LEO-MW data time and to re-map into the geographic coordinates both GEO-IR and LEO-MW measurements available observations. Note that, since spatial resolution of MW data is generally worse than IR ones, a MW field-of-view of nominal area A generally includes more than one IR pixel. As a result of the background process, a data set is generated, containing the per-pixel rain-rate retrieved from LEO-MW data, the co-located GEO-IR brightness temperature and the pixel geo-location. This process is continuously ongoing, since new LEO-MW and GEO-IR data are continuously ingested depending on available satellite platforms.

iii) The third step is a foreground process, started to derive the R-TIR inverse relationship once the data set has been updated. A pre-processing stage is accomplished after each background process. The IR retrieval relationships are updated every time a new set of combined data have been added to the data set relative to that subregion, and are derived using data archived in a time window of several hours (integration step). To assure that only the most recent rain history is captured and to guarantee a statistical significance of the training set, the R-TIR inverse relationship for a given sub-region is derived using only the most recent combined data.

iv) The last step is represented by the prediction of the surface rain-rate from IR measurements in a given subregion by applying the derived R-TIR algorithm (retrieval step). Many attempts have been carried out so far to derive this R-TIR relationship, using different techniques such as probability matching formulations, 


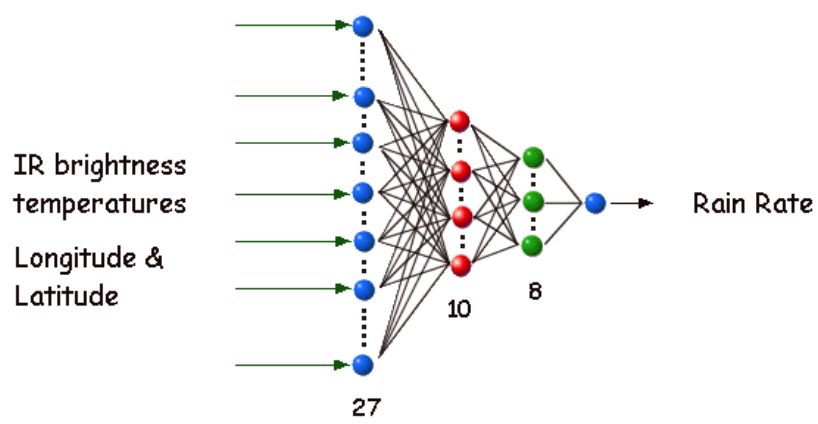

Fig. 2. Neural Network architecture for rainfall estimation.

regression methods and artificial neural networks. Details on the background and foreground processes are given Sect. 4.

\section{Neural rainfall estimation algorithm using microwave and infrared sensors}

The NEREMIS techniques shares the same methodology of MICRA, but uses a Multiply Layer Feed Forward NN architecture as shown in Fig. 2 (Grimes et al., 2003; Tapiador et al., 2004b). It is a four layers network using as input the IR TB from GEO-IR sensor and latitude and longitude information.

The NN is calibrated using input data derived from the GEO-IR sensor and output rain-rate retrieved from available LEO-MW measurements. This requires, as for MICRA, measurements from both IR and microwave sensors collocated in space and in time. The calibration period has been fixed for this study to $24 \mathrm{~h}$ and the validation has been carried on for the following day. In particular, the calibration periods are the $24 \mathrm{~h}$ of the 23 January 2003, while 24 January 2003 has been retained for the validation.

There is not any constraint in choosing a calibration period. The one day time step has been selected due to the length of the precipitation event that has been observed for at least tree days. In case of a short rain event the time step could be reduced to few hours.

The validation of NEREMIS has been carried on in the smaller area of central Italy due to data availability problem. The performances of the trained neural network will be shown in Sect. 4.

\section{Case studies and preliminary validation}

The MICRA technique has been tested on a few case studies characterized by intense and persistent rainfall over the Mediterranean basin. The LEO-MW data are provided by Special Sensor Microwave Imager (SSM/I) on board of the three Defense Meteorological Satellite Program (DMSP) platforms. LEO-IR data are provided by the Visible Infrared Spinning Scan Radiometer (VISSR) on board of the operational European geostationary satellite METEOSAT.
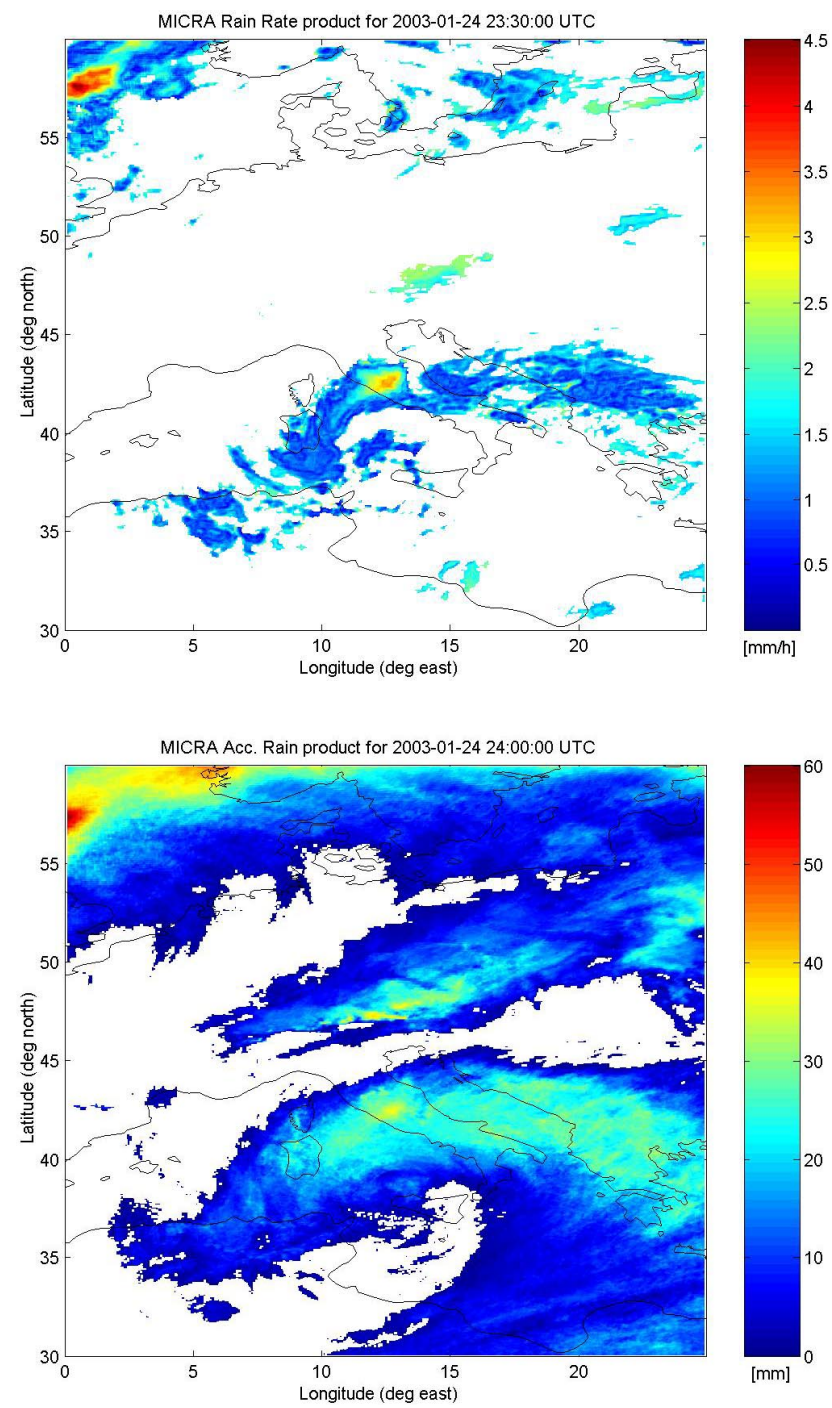

Fig. 3. Top: Rain rate estimated by MICRA for 24 January 2003, 23:30 UTC. Bottom: 24-hour accumulated rain as computed from 1-day time series of MICRA rain rate estimates for 24 January 2003.

The first case study we have analyzed occurred at the end of January 2003. In the top of Fig. 3 is shown rain rate over the Mediterranean basin as estimated by the current implementation of MICRA at 23:30 UTC of 24 January 2003. It is evident a large convective cell developing over Tuscany. Such a product is available every time a METEOSAT-VISSR image is released. By simply integrating a day-long time series of these estimated rain rates, we are able to provide 24 hour accumulated rain over the same area, as illustrated in the bottom of Fig. 3. In the $24 \mathrm{~h}$ of 24 January 2003, a large amount of rain has fallen near the costs of UK. The effects of the just mentioned convective activity over Tuscany are still visible.

For the same case study the NEREMIS estimates are shown. In Fig. 4 the instantaneous rain rate for 23:30 UTC and the $24 \mathrm{~h}$ accumulated rain are show for central Italy. Comparing Figs. 2 and 3 for the $24 \mathrm{~h}$ accumulated rain the 

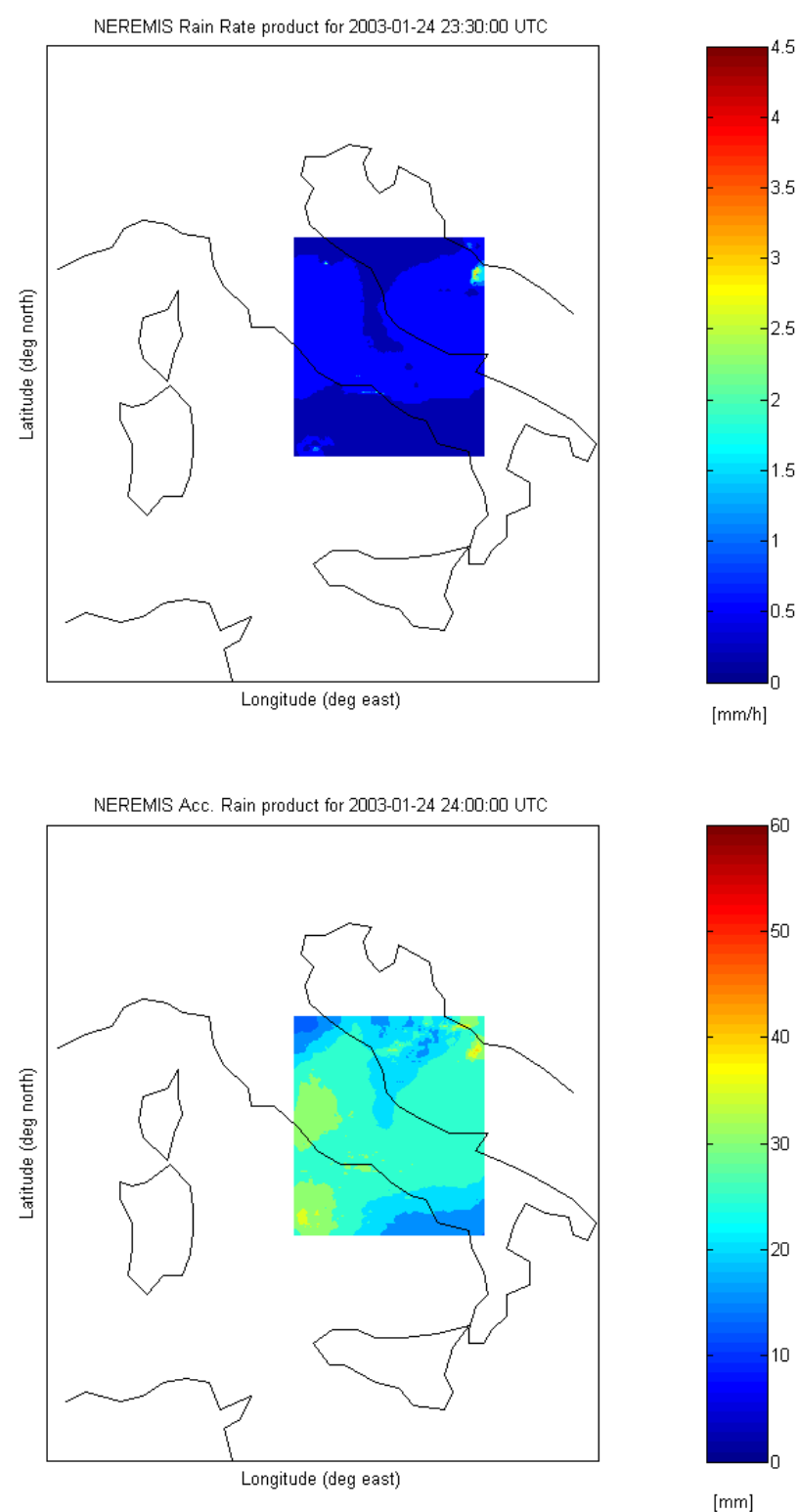

Fig. 4. Top: Rain rate estimated by NEREMIS for 24 January 2003, 23:30 UTC. Bottom: 24-hour accumulated rain as computed from 1-day time series of NEREMIS rain rate estimates for 24 January 2003.

shape and the intensity of the rain fields are in fairly good agreement, although the spatial domain is limited. The instantaneous rain rate field estimated for the 23:30 UTC by MICRA and NEREMIS show a similar shape, but the intensity is slightly different.

In the top panel of Fig. 5 is presented the 02:00 UTC product for the case study of 20 May 2003. Two areas with intense precipitation are distinguishable, the larger over Poland, while the other over Germany. A map of 24-hour accumulated rain for this case study is illustrated in the bottom of Fig. 5. As the meteorological situation has developed south-eastward, the largest amount of accumulated rain was found in Northern Italy. Indeed, flash floods were reported
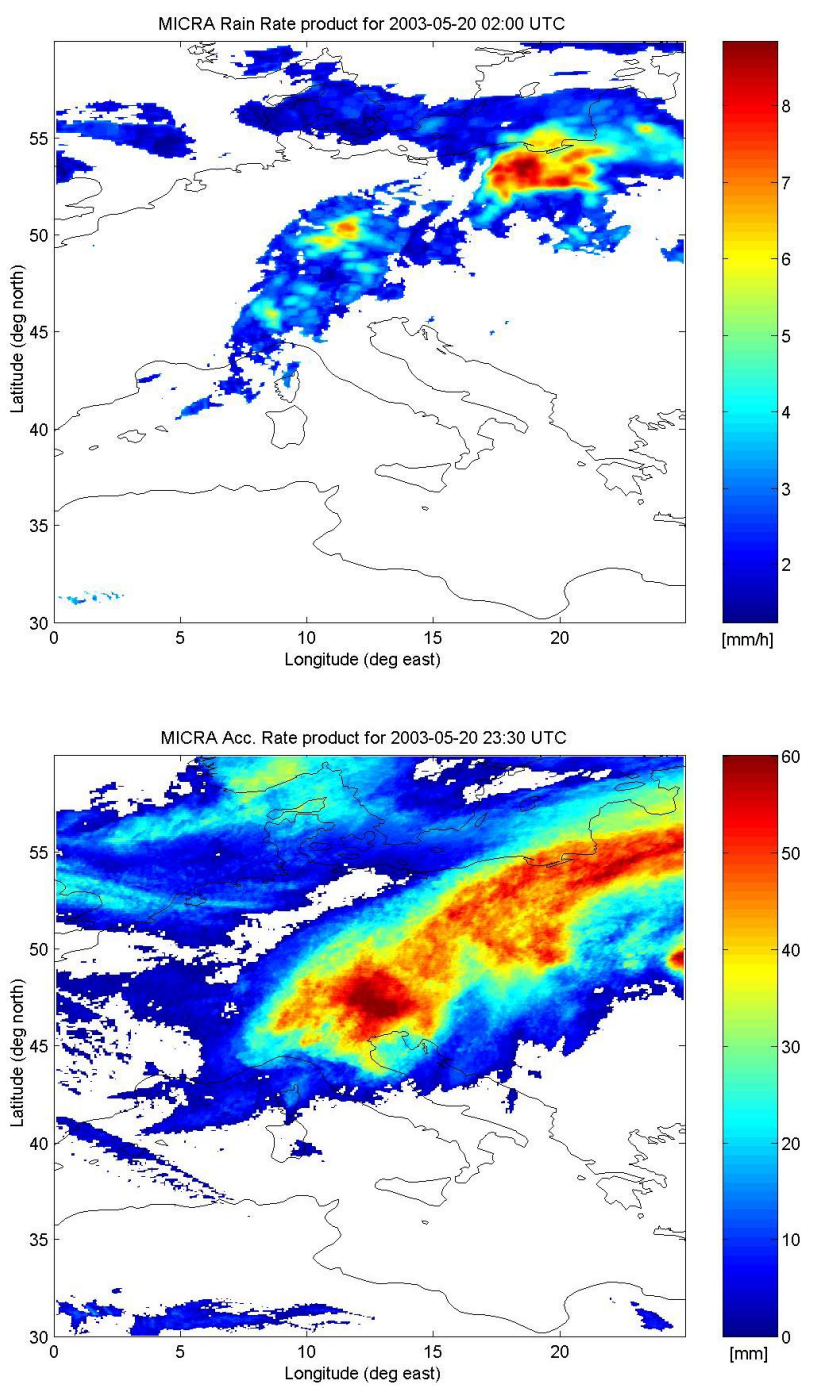

Fig. 5. Top: Rain rate estimated by MICRA for 20 May 2003, 02:00 UTC. Bottom: 24-hour accumulated rain as computed from 1-day time series of MICRA rain rate estimates for 20 May 2003.

near the cost of North-eastern Italy during 20-21 May 2003. Although qualitative results are encouraging, a systematic validation would be needed. A way to pursue this goal is by comparison with ground based remote (radar) and in situ (raingauge networks) measurements available for the areas under investigation, as illustrated in Fig. 6. Unfortunately, both data sets were not available during the presented case study.

A preliminary example of ground validation with radar measurements is presented in Fig. 7. Radar data (top) were collected by the S. Pietro Capofiume unit, owned by the Regional Environmental Agency of Emilia Romagna (ARPASMR), and processed by the Regional Meteo-hydro Service (SIM). At radar original resolution (order of $1 \mathrm{~km}$ ), differences in rate rates of one order of magnitude are found with respect to MICRA estimates (bottom). 


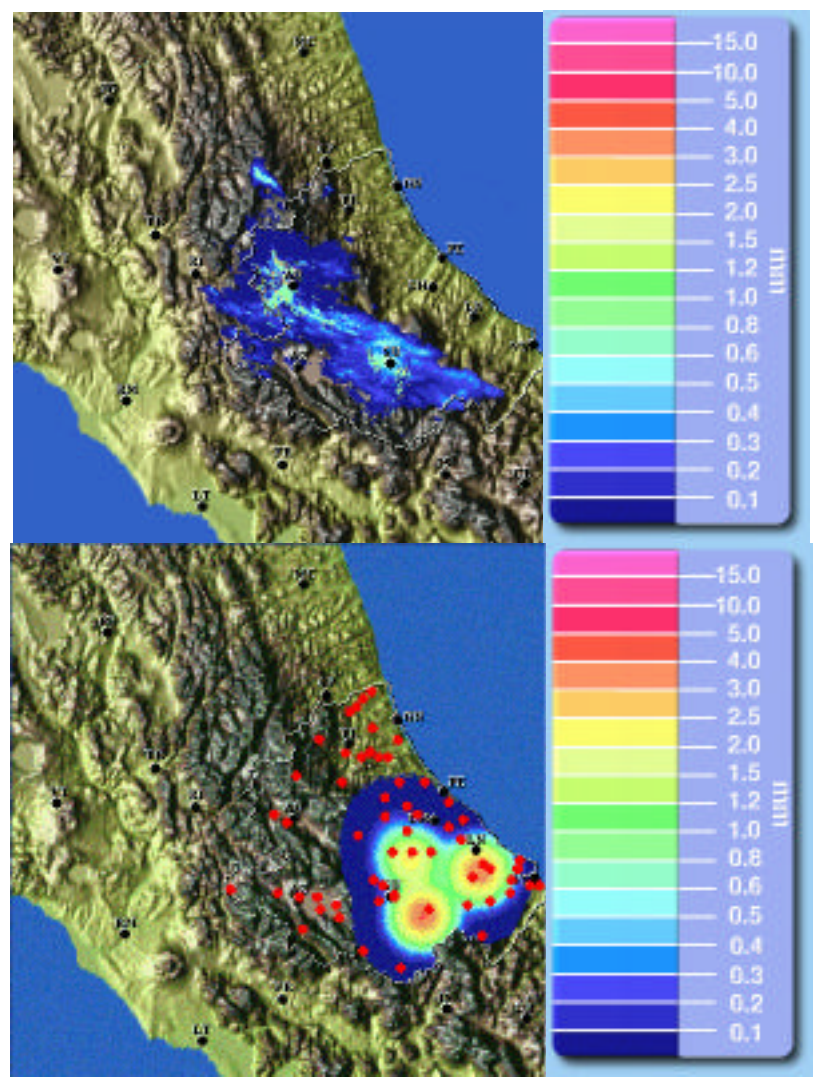

Fig. 6. Available measurements from ground-based radar and raingauge networks are used in the validation of MICRA.

However, a meaningful comparison has to take into account the fact that rain rate estimates by MICRA are limited by the relatively poor spatial resolution of both GEO-IR and LEO-MW measurements. Thus, radar spatial resolution needs to be degraded to the resolution achievable by satellite (order of $10 \mathrm{~km}$ ).

If we average radar measurements in Fig. 7 to match the satellite pixel resolution, and we consider only the area covered by the radar beam, we obtain what shown in Fig. 8. Now the qualitative comparison looks much better: areas of intense rain are fairly well detected and the difference of rain rate values is significantly reduced. Average errors are of the order of $30 \%$ with an overall trend to underestimate the radar estimates.

From these first results it seems that the present implementation of MICRA tends to underestimate rain rates values. Further analysis is needed in order to optimize the algorithm parameterization, such as choices of sub-region size, spacing and look-back time lag. Results for NEREMIS show a similar behavior.

\section{Summary and future developments}

The basic concepts of the MICRA technique have been introduced together with those of the $\mathrm{NN}$ based algorithm NEREMIS. A couple of case studies over the Mediterranean
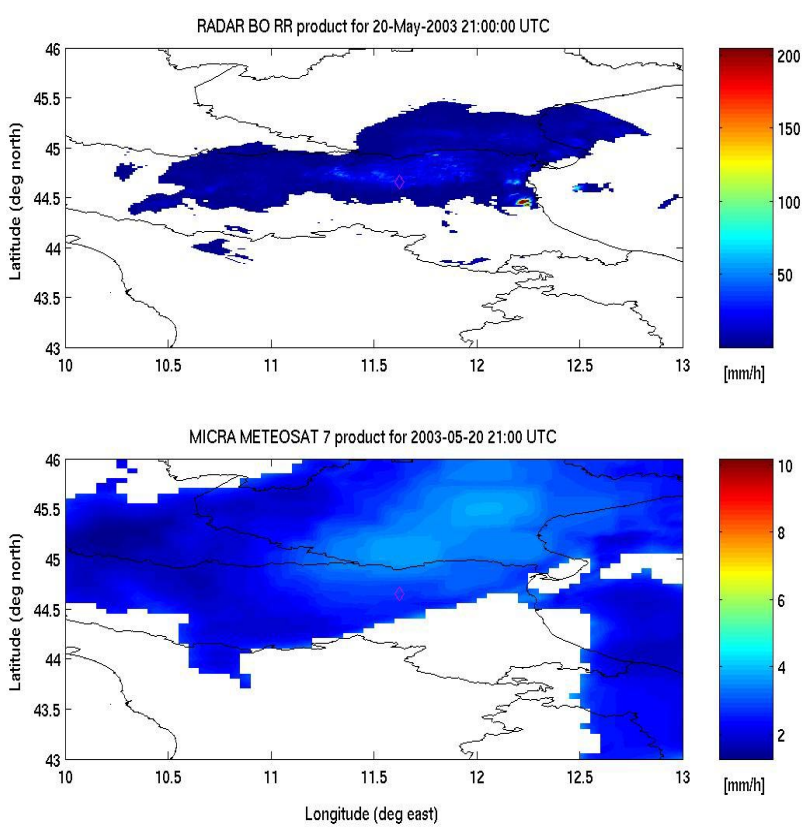

Fig. 7. A comparison between rain rates as measured by groundbased radar (top) and satellite-based MICRA technique (bottom). Original radar resolution, about $1 \mathrm{~km}$, is shown.
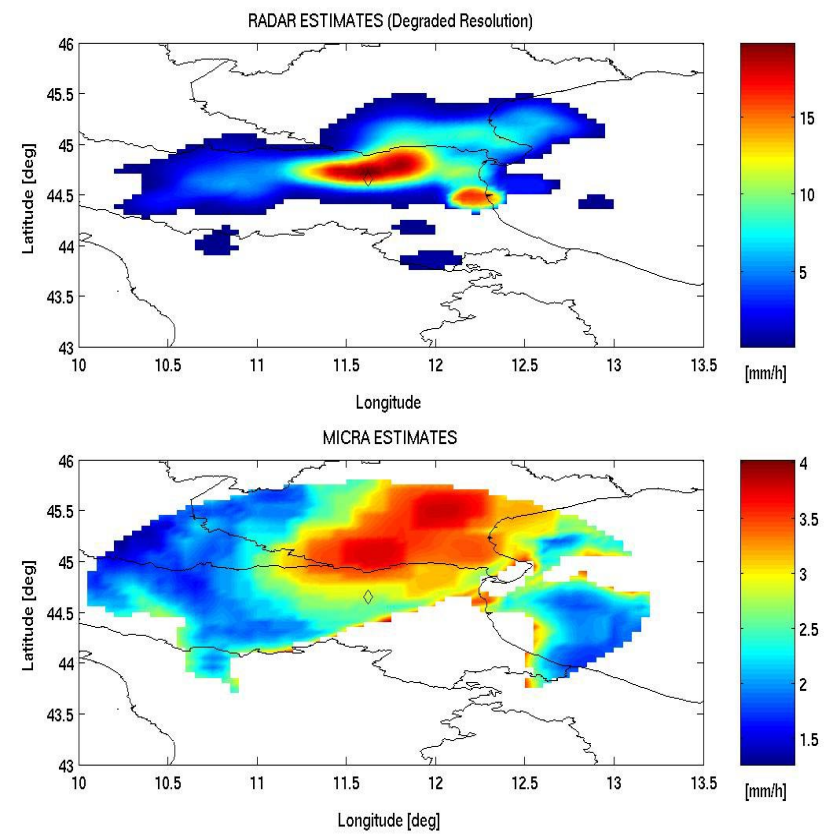

Fig. 8. Same comparison as Fig. 7, where radar data (top) have been averaged to match the satellite pixel resolution, and only the MICRA estimates within the area covered by the radar beam are considered (bottom).

basin have been discussed, through the analysis of rain rate and accumulated rain fields as estimated by MICRA and NEREMIS. In our first attempt of validation of the MICRA technique, we found encouraging results concerning the capability of the algorithm to detect the raining cells. 
From a quantitative point of view, MICRA seems to underestimate rain rate values when compared to ground based radar measurements. Possible sources of underestimation might be related to algorithm parameterization, such as the choice of sub-region size and spacing or the look-back time lag, whose optimum values have to be investigated in deeper detail.

A systematic validation of MICRA and NEREMIS with ground-based measurements is planned, but their intercomparison of estimated rain field shows encouraging agreements. Inclusions of data derived from other sensors, like MODIS and AMSR, are also foreseen.

Once that the algorithms have been tested and validated, the resulting optimized version will be included in a monitoring and forecast operational framework. The exploitation of Meteosat Second Generation - High Rate Information Transmission (HRIT) data is finally the challenging aim of a further development and extension of the proposed techniques.

Acknowledgements. This work has been partially funded by the EU Fifth-Framework EURAINSAT project (EVG2-2000-00522) and by EU Risk-AWARE project. Support by the Italian Space Agency (ASI), Ministry of Education and Research (MIUR) and by the GNDCI-CNR project is also acknowledged. Radar data were kindly provided by P. P. Alberoni (ARPA-SIM, Bologna, Italy).

Edited by: L. Ferraris

Reviewed by: C. M. Medaglia and another referee

\section{References}

Bellerby, T., Todd, M., Kniveton, D., and Kidd, C.: Rainfall Estimation from a Combination of TRMM Precipitation Radar and GOES Multispectral Satellite Imagery through the Use of an Artificial Neural Network, J. App. Met., 39, 2115-2128, 2000.

Ferraro R. R.: SSM/I derived global rainfall estimates for climatological applications, J. Geophys. Res., 102, 16 715-16735, 1997.

Grimes, D. I. F., Coppola, E., Verdecchia, M., and Visconti, G.: A neural network approach to real time rainfall estimation for Africa using satellite data, J. Hydrometeorology, 6, 4, 11191133, 2003

Hsu, K. L., Gao, X., Sorooshian, S., and Gupta, H. V.: Precipitation estimation from remotely sensed information using artificial neural networks, J. Appl. Meteor., 36, 1176-1190, 1997.
Kummerow, C. and Giglio, L.: A method for combining passive microwave and infrared rainfall observations, J. Atmos. Oceanic Tecnol., 12, 33-45, 1995.

Levizzani, V., Porcù, F., Marzano, F. S., Mugnai, A., Smith, E. A., and Prodi, F.: Investigating a SSM/I microwave algorithm to calibrate METEOSAT infrared instantaneous rain-rate estimates, Meteor. Applications, 3, 5-17, 1996.

Marzano, F. S., Palmacci, M., Cimini, D., Giuliani, G., and Turk, J. F.: Multivariate Statistical Integration of Satellite Infrared and Microwave Radiometric Measurements for Rainfall Retrieval at the Geostationary Scale, IEEE Trans. Geosci. Rem. Sens., 42, 4, 1018-1032, 2004.

Marzano, F. S., Giuliani, G., and Cimini, D.: Design of rapid update cycles of rain estimation: MICRA technique, 2nd-year Report of 5th-Framework EURAINSAT project, edited by: Levizzani, V., Jan 2003.

Marzano, F. S., Mugnai, A., and Turk, J.: Precipitation retrieval from spaceborne microwave radiometers and combined sensors, in: Remote sensing of atmosphere and ocean from space, edited by: Marzano, F. S. and Visconti, G., Kluwer Academic Publ., Dordrecht (NL), 107-126, 2002.

Marzano, F. S., Turk, J., Ciotti, P., Di Michele, S., and Pierdicca, N.: Potential of combined spaceborne microwave and infrared radiometry for near real-time rainfall attenuation monitoring along earth satellite, Int. J. Satell. Commun., 19, 4, 385-412, 2001.

Miller, S. W., Arkin, P. A., and Joyce, R.: A combined microwave infrared rain rate algorithm, Int. J. Remote Sensing, 22, 32853307, 2001.

Tapiador, F. J., Kidd, C., Levizzani, V., and Marzano, F. S.: A neural networks-based PMW-IR fusion technique to derive half hourly rainfall estimates at $0.1^{\circ}$ resolution, J. Appl. Meteor., 43, 576594, 2004a.

Tapiador, F. J., Kidd, C., Hsu, K. L., and Marzano, F. S.: Neural Networks in Satellite Rainfall Estimation, Meteorological Applications, 11, 1-9, 2004b.

Turk, J. F., Rohaly, G., Hawkins, J., Smith, E. A., Marzano, F. S., Mugnai, A., and Levizzani, V.: Meteorological applications of precipitation estimation from combined SSM/I, TRMM, and geostationary satellite data, in: Microwave Radiometry and Remote Sensing of the Environment, edited by: Pampaloni, P., VSP Intern. Sci. Publisher, Utrecht (NL), 353-363, 1999.

Vicente, G. A., Cofield, R. A., and Menzel, W. P.: The operational GOES infrared rainfall estimation technique, Bull. Amer. Meteor. Soc., 79, 1883-1898, 1998. 